\title{
Behavioural disturbance and visual hallucinations in a 78 year old man
}

\author{
David W Schultz, Graham G Lennox, James W Ironside, Charles P Warlow
}

\author{
Case presentation
}

This 78 year old widower was referred for hospital admission in late September with shortness of breath. He was an ex-smoker and had a past history of chronic obstructive airways disease and ischaemic heart disease, having had a myocardial infarction 12 years earlier. He was experiencing weekly attacks of angina at rest and with exertion. His regular medications included bronchodilators, diuretics, and digoxin, and he had also recently been started on a course of prednisolone with omeprazole cover, for a presumed exacerbation of chronic obstructive airways disease.

On examination, he was apyrexial and not short of breath at rest. Although having evidence of chronic obstructive airways disease, there was no evidence of any acute respiratory compromise. He was in atrial fibrillation at a rate of $80 /$ minute and had an aortic systolic murmur but no evidence of cardiac failure. No neurological abnormality was noted.

Shortness of breath did not seem to be a significant problem in hospital. Blood gases disclosed mild hypoxaemia and hypercapnia on air similar to previous recordings, and echocardiography confirmed moderate aortic stenosis with normal left ventricular function. However, the sheltered accomodation from which he was referred refused to accept him back. It then became apparent that the main reason for his referral to hospital was because of concern about his recent behaviour at home. He had been living in a warden controlled residence for the previous 6 months, and had recently become disruptive, especially at night.

He often became anxious and called out the warden or his general practitioner (GP) many times each week. A similar pattern of behaviour was noted during his hospital stay, often needing reassurance at night. He had seen psychiatrists intermittently during the past 5 years. When he was first seen, he was assessed as having a prolonged grief reaction after his wife's sudden death 12 months earlier. $\mathrm{He}$ was reviewed again 4 years later, at which stage he was considered to have a reactive depression and was begun on the antidepressant sertraline. He had again been referred by his GP in the month before the present admission after developing visual hallucinations, coinciding with the recent withdrawal of his antidepres- sant drugs. These usually occurred at night and involved women, one of whom he identified as his late wife. He had insight into this problem, and also admitted to feeling disoriented on occasions. He had been experiencing floaters in both eyes for about a year, in association with a gradual deterioration of his vision. He thought that his memory had been deteriorating, and he often mislaid objects around the house. On mental state examination he had a mild global impairment affecting attention, short term memory, calculation, construction, and knowledge of current affairs. It was considered that his general ill health, polypharmacy, and probable eye pathology were contributing to his visual hallucinations, and that he had a mild depression for which he was begun on sulpiride. Little change was noted at outpatient review 1 month later.

In view of the concerns raised by the warden he was again reviewed by the psychiatrists while an inpatient for his breathing problems. They noted a significant decline physically, and considered that he had an agitated depression, being upset at the prospect of losing his accomodation. His antidepressant was changed to trazodone.

During a home visit in early October, it was clear that he was disorientated and unsafe in his own kitchen, with no insight into his problems. $\mathrm{He}$ was unable to read the cooker dials and would assess which one was on by touching the rings until he found the hot one! He became increasingly disruptive on the ward, wandering and falling occasionally, and continuing to call out, requiring intermittent thioridazine to settle. He developed urinary and faecal incontinence, but with no evidence of a urinary tract infection. He became markedly confused and was noted to have a shuffling gait, after which the thioridazine was stopped. His upgaze was poor, but there were no other focal neurological signs. His blood screens remained normal except for mild hypercapnia $\left(\mathrm{p}_{\mathrm{a}} \mathrm{CO}_{2} 7.4 \mathrm{kpa}\right)$ as noted on admission. A brain $\mathrm{CT}$ was attempted, but was unsuccessful as he would not lie still. Courses of augmentin and ceftriaxone were given although no focus of infection was identified.

By late October he had become increasingly withdrawn and developed a resting hand in revised form

9 July 1998

Accepted 25 July 1998 
tremor not thought to be typically parkinsonian. He would open his eyes but tended to stare ahead, and he no longer spoke. His oral intake was poor and he became dehydrated, developing a raised plasma sodium of 163 $\mathrm{mmol} / \mathrm{l}$ (normal 135-145 $\mathrm{mmol} / \mathrm{l}$ ) and renal failure, with a urea concentration of 54.8 $\mathrm{mmol} / 1$ (normal 3.3-6.6 mmol/1) and a creatinine concentration of $319 \mu \mathrm{mol} / 1$ (normal $70-110 \mu \mathrm{mol} / \mathrm{l})$.

An EEG disclosed a widespread patchy disturbance, with intermixed non-specific transient wave forms and more overt epileptiform sharp waves. "Twitches" of the upper limbs were noted during the recording. Correlation between the "twitches" and any electrocerebral disturbance was generally poor; however, on occasion there was coincidence of the EEG and clinical change (cortical myoclonus).

At this stage he was first reviewed by a neurologist, who thought that he was cortically blind, but could find no other focal neurological features. There were no myoclonic jerks or frontal lobe reflexes demonstrable.

$\mathrm{He}$ continued to deteriorate and at the request of the family was begun on a diamorphine infusion to alleviate any distress. He died 24 hours later. Consent was obtained for a limited postmortem examination.

\section{Discussion}

Dr G Lennox

Charles Warlow asked me to discuss this case as if it was a normal Friday afternoon referral from the medical wards. He made this easy! As in most such consultations, we have very little history, the patient was not properly examined at the relevant time, and the results of crucial investigations were unavailable. We are, however, told that the patient was a 78 year old man. At least this means that we do not have to worry about rare leukodystrophies and storage diseases.

There are two possible scenorios in relation to his neurological disease. Either this was a very nasty illness which started at the end of September and caused him to die at the beginning of November, or it was a more slowly progressive illness with a long psychiatric prodrome.

Five years before his admission he had what was thought to be a prolonged grief reaction or a reactive depression, but did not respond to treatment. Even at the beginning of the year of his admission, 9 months before he died, the symptoms were largely behavioural; he was anxious and disruptive, and kept calling out his warden and his GP. Then, in the month before admission, he developed complex formed visual hallucinations. We are told that his eyesight was poor. Complex visual hallucinosis in the presence of poor visual acuity is known as the Charles Bonnet syndrome. Recently it has become clear that visual hallucinations are particularly severe in dementing illnesses when the visual acuity is poor.

Whatever interpretation is made of these earlier behavioural symptoms, it is quite clear that in the last couple of months of this man's illness there really was a very rapid decline. We get some vital information in the window of opportunity before he started to go downhill so quickly when he underwent a cognitive examination. We are told that there was mild global impairment affecting attention, short term memory, calculation, construction, and knowledge of current affairs. This is immensely helpful as it tells us something of the anatomy of the disorder. There was involvement of the posterior hemispheres with the problems with calculation and construction. Anterior executive functions and attention were also affected. Indeed as the illness progresses there are other behavioural suggestions of both anterior and posterior dysfunction. He cannot recognise the controls on his cooker (which could be interpreted as visual agnosia), but he has no insight into the fact that he is unsafe (which suggests frontal impairment).

He then became confused and agitated, and was put on thioridazine. He became incontinent of urine and faeces. He developed a shuffling gait and later a resting tremor; this raises the possibility of parkinsonism, perhaps unmasked by the neuroleptic drug. The only other neurological finding at that stage was impaired upwards gaze, which is unremarkable at the age of 78 years but also occurs in a wide range of degenerative diseases of the nervous system including Alzheimer's disease, Parkinson's disease, dementia with Lewy bodies, Steele-Richardson syndrome, corticobasal degeneration, and Creutzfeldt-Jakob disease. A vertical gaze palsy also occurs with hydrocephalus or tumours of the pineal region (as part of Parinaud's syndrome).

Later he became mute and twitchy. He had an EEG which showed generalised slowing, and some discharges which seemed to correlate with the twitches suggesting that they were due to cortical myoclonus. This is again a nonspecific feature. One sees myoclonus in all of the cortical degenerations, and in any event he was metabolically deranged, with carbon dioxide retention and uraemia which can both cause myoclonic jerks. He was finally examined by a neurologist who thought he was cortically blind.

In summary, we have a 9 month history of a dementing illness with or without a 5 year history of a prodromal psychiatric illness. The dementia was characterised initially by anxiety and visual hallucinations, then a period of agitation and confusion, and then gait disturbance and tremor which may or may not have been induced by drugs. He rapidly developed myoclonic jerks, became definitely mute, probably blind, and died.

In dementia, just as in any other neurological problem, the history is of paramount importance. You hope to obtain information that will give you some idea of the overall pattern of the illness, and a clue to the likely type and distribution of the pathology. The range of neurological symptoms, the presence or absence of additional non-neurological symptoms, and whether the patient has insight into the problem or not, are all extremely important. A family history may be helpful: we increasingly recognise that many neurodegenerative diseases have a 
genetic basis. The examination must test a range of cognitive domains, including memory, attention, language, praxis, visuospatial abilities, and frontal lobe function, to discover which brain regions are affected. We need to establish if the cognitive deficit is focal or widespread, whether it affects mainly temporoparietal functions or mainly frontal functions, and so on. This assessment is supplemented by the conventional neurological examination. Additional specific neurological features that have diagnostic value include abnormal movements, upper or lower motor neuron signs, and peripheral neuropathy. Finally the examination must include an assessment of mental state, with depression representing an important and potentially treatable element in many dementias. ${ }^{2}$

In this case, the history is a little vague, there are no specific physical signs, and the differential diagnosis is broad. We must start by considering the causes of a rapidly progressive dementia, before moving on to consider the possible relevance of the prodromal psychiatric features. I am assuming that the screening tests excluded a metabolic or toxic encephalopathy, including (given his visual complaints), digoxin intoxication, which can produce yellow vision.

The next thing to consider is a malignant process, particularly a poorly localisable malignancy such as a glioma arising in the corpus callosum and spreading out into the cerebral hemispheres as a butterfly lesion, and multifocal tumours such as metastases and primary cerebral lymphoma. These must be excluded by imaging where possible. In any rapidly progressive neurological disorder, the possibility of a paraneoplastic syndrome must be considered. Limbic encephalitis generally causes amnesia, usually swiftly followed by brainstem signs (which were not noted here).

We know that this patient had multiple vascular risk factors. If this had been a one off illness or a stuttering illness, in which the patient went into this state over the course of a week rather than a couple of months, the possibility of basilar thrombosis would have to be considered, giving a top of the basilar syndrome. These patients, because they can infarct deep diencephalic structures, may become drowsy and irritable or even frankly delirious and, unlike most acute confusional states, this can go on for weeks. Visual field defects are common, but usually give rise to a hemianopia rather than cortical blindness. Against this diagnosis are the duration of the history and the lack of additional brainstem and pupillary signs such as skew deviation and distorted, teardrop shaped pupils.

A more likely possibility is multi-infarct dementia, due to either multiple emboli from the heart or multiple strokes in the carotid territory. The early mild global pattern of cognitive impairment makes a succession of large infarcts relatively unlikely. Indeed, it would seem that this patient's decline was relatively smooth and relentless rather than stepwise. We are also told that there were no focal signs at any stage so I think that we can be relatively confident in ruling out multiple large vessel infarcts. The same argument applies to haemorrhagic lesions from amyloid angiopathy, although this can occasionally present as a dementia. More difficult to exclude is subcortical small vessel arteriosclerosis, traditionally termed Binswanger's disease, because this can present as a smoothly progressive disorder. These patients typically present with the so-called clinical triad of normal pressure hydrocephalus (frontal cognitive impairment, chaotic gait disturbance, and incontinence of urine). Binswanger's disease is possible but does not fully explain the global nature of this patient's deficits, nor the very rapid progression. The same arguments apply to cerebral vasculitis and, furthermore, there are no positive features of this group of disorders, such as headache or any systemic features. Finally, subdural haematoma is extremely unlikely with the preceding evidence of widespread bilateral cognitive impairment, before the patient started to deteriorate rapidly, but should be excluded by appropriate imaging.

Next we must consider infections. He was elderly and taking steroids, which would make him more vulnerable to unusual infections. Progressive multifocal leucoencepalopathy could account for a dementia with cortical blindness, although usually there are other focal signs at some point along the line, and usually the patient goes through a phase of being hemianopic before losing vision altogether. Opportunistic infections, HIV, and the more chronic meningitic illnesses are statistically unlikely. Whipple's disease is statistically very unlikely; indeed, this is a disease which only occurs in clinicopathological conferences. If you ever need to diagnose Whipple's disease, just ask yourself "Am I in a clinicopathalogical conference?" and if you are not you can relax. Even here there are things which are against it being Whipple's disease; there are no systemic features such as arthralgia or malabsorption. The patient does not have the characteristic (but not pathognomic) neurological manifestation in which the eyes and jaw move simultaneously, so-called oculomasticatory myorhythmia. Finally, the patient has been treated blindly with antibiotics, including cephalosporins, which would probably have killed off the Whipple's organism if this was Whipple's disease. Similarly, there are no positive signs of neurosyphilis.

We must next turn to a disease which we should perhaps categorise as of uncertain aetiology-Creutzfeldt-Jakob disease (CJD). I am sure this is a diagnosis which has occurred to many people in the audience here in Edinburgh. The prominent early anxiety is not just a feature of the new variant form of CJD and occurs in some patients with conventional sporadic CJD. The rapid progression, if it truly was a very short illness, would also be consistent with CJD, as indeed would be the development of a movement disorder. Extrapyramidal features are relatively common in prion dementia, although I think a true resting tremor would be a very unusual manifestation. The myoclonus would be consistent with CJD, but it was rather evanescent here. The EEG would certainly be consistent with early CJD, but does 
not show the classic picture of triphasic complexes. Finally, if he was truly cortically blind by the end of his illness, this would also be consistent with CJD. But it must be remembered that CJD remains a rare disease, particularly in very old people like this. There are also several hints that the history may be longer than the dramatic final few months that are described in detail. The longer the history, the less likely this is to be CJD.

Finally, while we are dealing with rapidly progressing disorders I want to mention dementia with Lewy bodies. This is the current consensus term for a disease which has been described under a range of different rubrics such as Lewy body dementia, diffuse Lewy body disease, or cortical Lewy body disease. Although this is normally a slowly progressive dementing illness with a tempo similar to that of Alzheimer's disease, it can occasionally present in a very fulminant and aggressive way. ${ }^{34}$ Such patients often subsequently plateau out and stabilise for a few months before they die.

Returning to the possibility that the history is longer than a few months, the relevance of the neuropsychiatric prodrome must be considered. This raises the possibility of a frontotemporal dementia. This is a syndrome with a wide range of different pathologies, including processes which resemble motor neuron disease and corticobasal degeneration (despite the absence of the usual clinical manifestations of these disorders) and classic Pick's disease pathology (although this is rare).$^{56}$ Frontotemporal dementia is a syndrome which starts with behavioural change, agitation, anxiety, and endless trips to the GP with multiple somatic complaints before it becomes obvious that there is cognitive impairment and before the patient's behavioural repertoire shrinks down to a range of stereotyped behaviours, stereotyped utterances, and finally a mute state. The hallmark of frontotemporal dementia, however, is that the posterior functions are spared and in particular visuospatial functions are normal. This effectively excludes it from consideration here. Again, dementia with Lewy bodies will sometimes present with very hypochondriacal features. This is a reflection of frontal predominance of pathology and so when Alzheimer's disease happens to involve the frontal lobe particularly severely it too can present in the same way.

Turning to the visual hallucinations, these do seem to be statistically most common in dementia with Lewy bodies. You are perhaps beginning to see which way I am thinking. His "persistent confusional state" may also provide a clue. We do not truly know if this was confusion as we are not told enough about the phenomenology, but it would seem likely that there have been at least some episodes of nocturnal confusion. Obviously intercurrent medical problems which may well have been operative here have to be born in mind, but patients with dementia with Lewy bodies commonly have recurrent episodes of confusion for which intercurrent medical problems are never found. These are patients who end up with very thick casenotes full of negative urine and sputum cultures, and negative searches for cerebrovascular disease.

Finally, the visual agnosia must be considered. As an early symptom, this raises the possibility of the focal posterior dementia syndromes. These are much rarer than frontal syndromes, but have broadly speaking the same pathological differential diagnosis. As already noted, this symptom excludes frontotemporal dementias, but none of the other degenerative conditions. It is perfectly compatible with Alzheimer's disease, multi-infarct dementia of any kind, dementia with Lewy bodies, and CJD.

So my differential diagnosis is starting to become clear. I am seriously considering CJD and I would want to clarify the duration of history if I possibly can. Nothing so far excludes Alzheimer's disease which is far and away the commonest degenerative dementia, but the constellation of features that I have described would make that an unusual pathological diagnosis. So, finally, I return to dementia with Lewy bodies. The current consensus criteria for the diagnosis of probable and possible dementia with Lewy bodies have recently been published. ${ }^{7}$ These require a dementia syndrome of the kind seen here, together with at least two out of (1) fluctuating cognition, (2) recurrent visual hallucinations, and (3) spontaneous parkinsonism. This patient seems to have had the first and second of these; his parkinsonism is also consistent with the diagnosis, but does not add diagnostic weight because it followed neuroleptic therapy and was not disproportionate (there was no "neuroleptic sensitivity"). I personally have never seen a patient with dementia with Lewy bodies who I was certain was completely cortically blind, but that might just be because I do not routinely examine people shortly before they die.

In conclusion, I think that the primary pathological diagnosis will be dementia with Lewy bodies. As this man is 78 years old he will also have some plaques and very probably some tangles but I personally would not regard

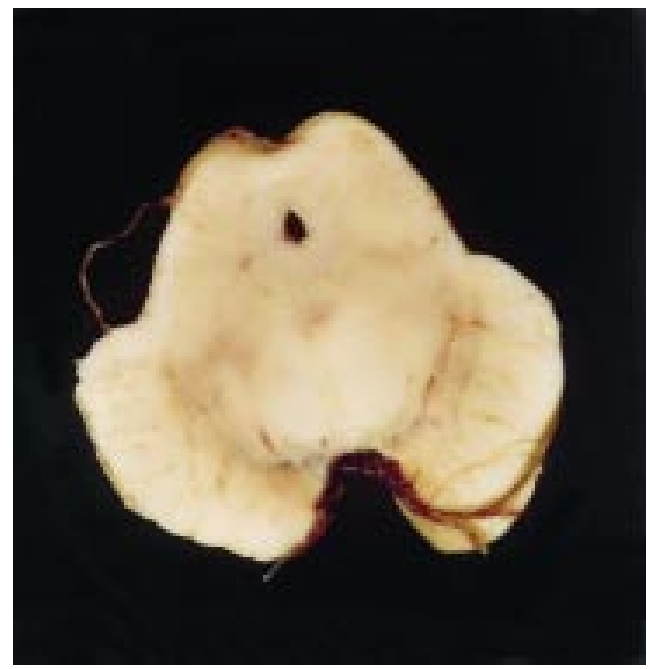

Figure 1 The substantia nigra in the midbrain shows a marked loss of pigment with a yellow/grey discolouration on macroscopic examination. 
Alzheimer's disease as the likely cause of his problem. Because of his age and his multiple vascular risk factors he may well have a little bit

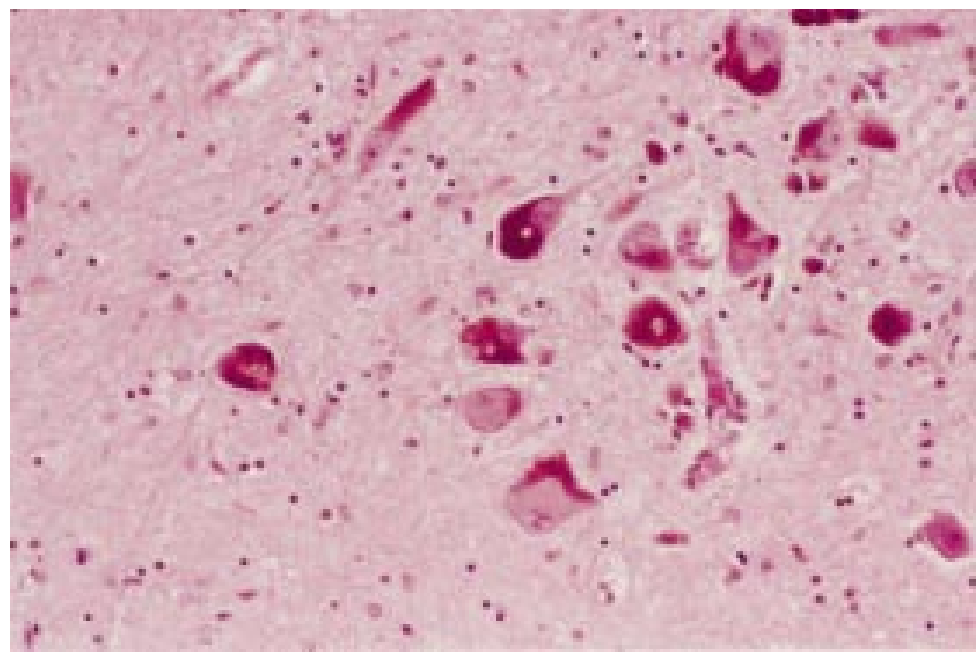

Figure 2 Histological examination of the substantia nigra shows numerous Lewy bodies in surviving pigmented neurons, with multiple inclusions present within some cells (centre).

The adjacent tissue is markedly gliotic. Haematoxylin and eosin stain.

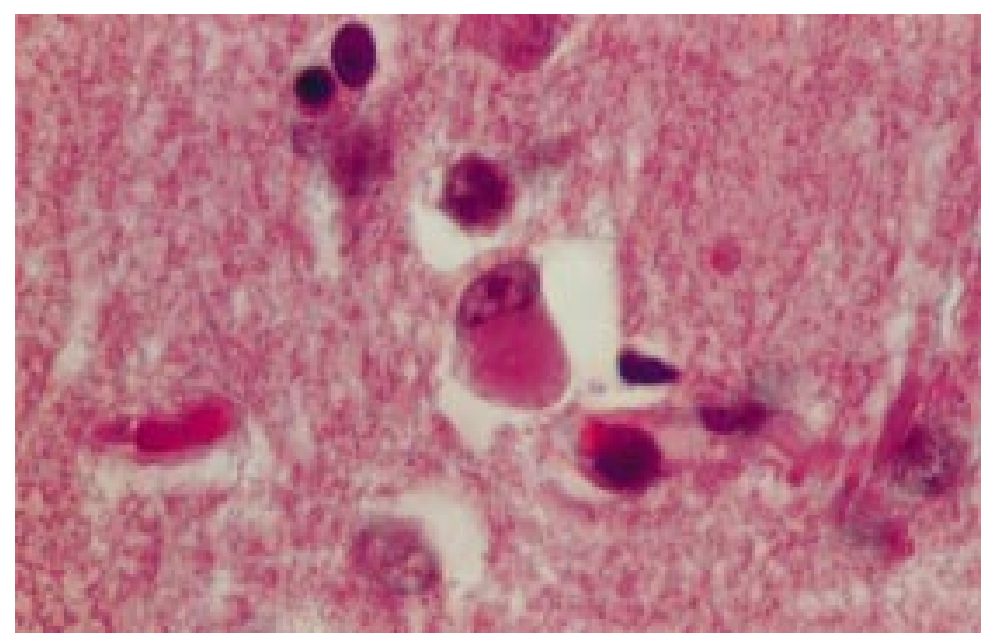

Figure 3 An occasional neuron in the anterior cingulate gyrus contains an ill defined globose homogenous eosinophilic inclusion characteristic of a cortical Lewy body (centre). Haematoxylin and eosin stain.

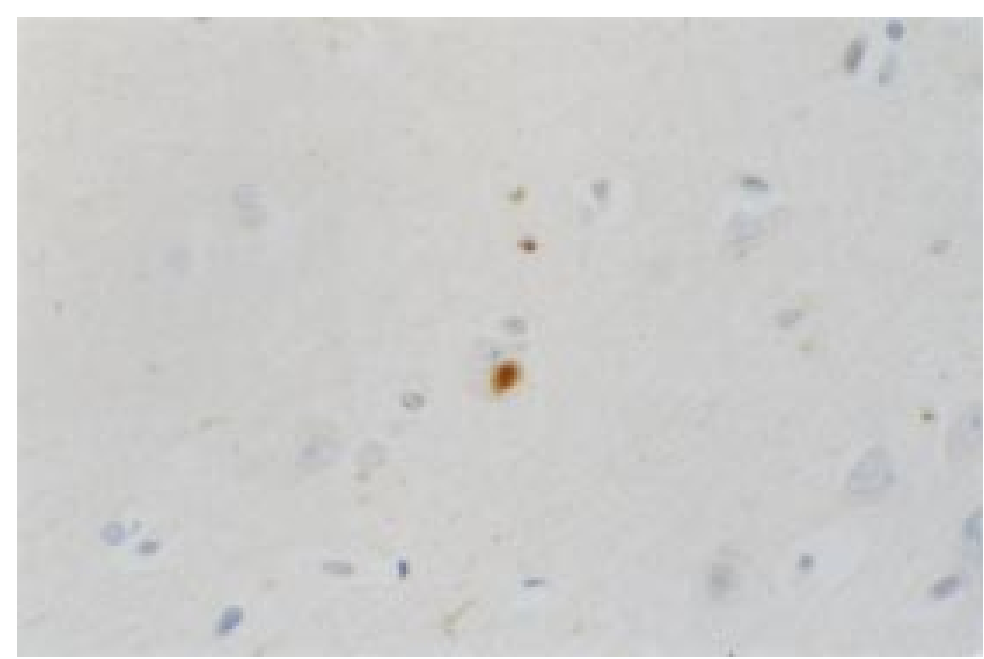

Figure 4 Ubiquitin immunocytochemistry shows strong brown positive staining of a cortical Lewy body within the cingulate gyrus (centre). Occasional positively stained dot-like structures are present within the adjacent neuropil, possibly representing small neuritic processes. of incidental mild vascular disease, but I'm not hedging my bets; the diagnosis here is dementia with Lewy bodies.

\section{Dr $\mathcal{F} W$ Ironside}

The clinical diagnosis at the top of the list in our hospital was CJD, which is why I was asked to examine the brain. The brain was fixed and weighed $1190 \mathrm{~g}$. On cross section there was a mild degree of cortical atrophy with accompanying ventricular dilatation, but nothing too abnormal for the patient's age. There were some prominent perivascular spaces in the basal ganglia but nothing to suggest infarction or haemorrhage. There was no diffuse white matter disease, no sign of progressive multifocal leucoencephalopathy, or any other focal lesions, nor evidence of a glioma, lymphoma, or any sort of tumour. There was nothing abnormal in the meninges, no haemorrhages, and no evidence of vasculitis. The cerebellum was largely normal and certainly not atrophied, but in one area there was a little blurring of the grey-white matter outline to make me wonder if perhaps there was an area of ischaemic damage, not quite in a typical vascular boundary zone. In the midbrain, the substantia nigra was pale (fig 1) and dissection down to the pons showed the locus coeruleus to be poorly defined, suggesting the possibility of Lewy body disease.

In the discoloured area of the cerebellar cortex, when examined microscopically, there was evidence of ischaemic damage with quite a marked loss of Purkinje cells, reactive gliosis, and some granular cell loss. This ischaemic damage in the cerebellum was not recent, and there was no extensive infarction in the cerebellum or brainstem. In the substantia nigra, Lewy bodies were indeed present on microscopy (fig 2), with multiple inclusions in some cells and pigment accumulation in macrophages. The locus coeruleus histologically also contained Lewy bodies, which suggests the diagnosis of Parkinson's disease. Ubiquitin immunocytochemistry on the substantia nigra shows how well these inclusions stain with this particular antibody. If it is appropriate to look for cortical Lewy bodies, this is the method of choice after the experience from Nottingham. ${ }^{8}$ In the cerebrum, there was cell loss in the hippocampus, but only to a mild degree. In routinely stained sections of the cortex the cores of a few plaques were identified. These were not prion plaques but the $\mathrm{A} \beta$ plaques occurring in Alzheimer's disease. On immunocytochemistry neurites were evident throughout these plaques, and a few of the surviving pyramidal neurons in the hippocampus contained neurofibrillary tangles. These tangles were not particularly excessive in relation to the patient's age and certainly using the established CERAD (Consortium to Establish a Registry for Alzheimer's Disease) criteria, ${ }^{9}$ a diagnosis of Alzheimer's disease would not be made. Immunocytochemistry for the $A \beta$ protein of Alzheimer's disease again showed quite a lot of plaques, often rather diffuse in structure, within the temporal and frontal cortex. A few larger plaques were present in layers 
3 and 4, but there really was no convincing evidence of amyloid angiopathy. The white matter was unremarkable with no evidence of demyelination and no inflammation.

Looking in greater detail at the cortex, it is necessary to establish whether or not there are Lewy bodies in the cortical neurons. On routine stains it was often rather difficult to find a convincing example of these inclusions, but a few were identified in the anterior cingulate gyrus (fig 3). Immunocytochemistry for ubiquitin stained these inclusions quite strongly (fig 4), and in some areas you can see that there were actually multiple inclusions within the anterior cingulate and temporal cortex. In relation to the clinical possibility of cortical blindness, the occipital lobe was not severely involved, and so I cannot provide a good pathological correlation in the absence of any other abnormalities in the visual cortex and related areas.

So, to summarise, I have to agree that the diagnosis is dementia with Lewy bodies. The relation between Lewy bodies and $\mathrm{A} \beta$ plaques is of considerable current interest. ${ }^{10}$ Additionally there is evidence of mild cerebrovascular disease, which is unlikely to have contributed significantly to the final illness.

\section{Comment}

Dr G Lennox

The key to the diagnosis of dementia with Lewy bodies is to consider it in patients with dementia and any degree of parkinsonism, particularly if there are features of fluctuating cognition, visual hallucinations, and a psychiatric prodrome. The teaching message of this case is it can present in an aggressive and fulminant way. The interrelation with Alzheimer type pathology is extremely interesting and probably reflects the inadequacy of disease labels to describe a complex situation where a mesh of different genetic risk factors probably acts to generate the different types of neuropathology.

The case was presented in this form as a clinicopathological conference at the Edinburgh Advanced Neurology Course, 28 March 1996.

1 McShane R, Gedling K, Reading M, et al. Prospective study of relations between cortical Lewy bodies, poor eyesight, and hallucinations in Alzheimer's disease. 7 Neurol Neurosurg Psychiatry 1995;59:185-8.

2 Goodwin GM. Functional imaging, affective disorder and dementia. Br Med Bull 1996;52:495-12.

3 Byrne EJ, Lennox G, Lowe J, et al. Diffuse Lewy body Byrne EJ, Lennox G, Lowe J, et al. Diffuse Lewy body
disease: clinical features in fifteen cases. $\mathcal{f}$ Neurol Neurosurg disease: clinical features in fifte
Psychiatry 1989;52:709-17.

4 Lennox G. Lewy body dementia. In:Rossor $M N$, ed. Unusual dementias. Balliere's Clinical Neurology 1992;1:65376.

5 Jackson M, Lowe J. The new neuropathology of degenerative frontotemporal dementias. Acta Neuropathol (Berl) 1996;91:127-34

6 Cooper PN, Jackson M, Lennox G, et al. Tau, ubiquitin and alphaB-crystallin define the principal causes of degenerative frontotemporal dementia. Arch Neurol 1995;52:101115

7 McKeith IG, Galasko D, Kosaka K, et al. Consensus guidelines for the clinical and pathological diagnosis of dementia with Lewy bodies (DLB): report of the consortium on
DLB international workshop. Neurology 1996;47:1113-24.

8 Lennox G, Lowe J, Landon M, et al. Diffuse Lewy body disease: correlative neuropathology using anti-ubiquitin immunocytochemistry. $₹$ Neurol Neurosurg Psychiatry 1989; 52:1236-47.

9 Mirra SS, Heyman A, McKeel D, et al. The Consortium to Establish a Registry for Alzheimer's Disease (CERAD). Establish a Registry for Alzheimer's Disease (CERAD).
Part II. Standardisation of the neuropathologic assessment of Alzheimer's disease. Neurology 1991;41:479-86.

10 Gibb WRG, Mountjoy CQ, Mann DMA, et al. A pathological study of the association between Lewy body disease and Alzheimer's disease. $\mathcal{F}$ Neurol Neurosurg Psychiatry 1989;52: $701-8$. 